\title{
A importância dos registros de câncer de base populacional para identificar casos de câncer ginecológico relacionados com câncer colorretal em população de risco*
}

\author{
The importance of population-based cancer registry to identify the cases of \\ gynecologic cancer related with the colorectal cancer in population at risk
}

\author{
Nazira Mahayri' e Djalma de Carvalho Moreira Filho²
}

\begin{abstract}
Resumo
O s registros de câncer de base populacional são uma fonte importante de casos para os estudos da epidemiologia e prevenção de câncer. 0 câncer colorretal é a segunda causa de morte por neoplasias em mulheres nos Estados U nidos. Weinberg et al ${ }^{8}$ através do estudo de 101.734 casos de cânceres de endométrio, colo do útero e ovário registrados no programa de vigilância e epidemiologia dos Estados U nidos (SEER), demonstrou 0 aumento do risco relativo (RR) de câncer colorretal seguindo-se o câncer de ovário $(R R=3,7)$ e de endométrio $(R R=3,4)$. 0 Registro de Câncer de Base Populacional de Campinas-São Paulo, a partir de 6.539 casos registrados de neoplasias malignas incidentes no município de Campinas no período de 1991 a 1995, utilizou para identificar os casos de câncer ginecológico relacionados com câncer colorretal e vice-versa nesta base de dados, um procedimento metodológico semelhante ao estudo mencionado, e reuniu 810 casos de mulheres com diagnóstico de câncer do colo uterino (265), endométrio (178), ovário (97), cólon e reto (270). N esta casuística, a média de idade para 0 câncer de colo do útero foi de 53,6 anos; endométrio 62,2; ovário 53,9 e colorretal 61,9 anos. Após a comparação de todos os casos selecionados, identificaram-se duas pacientes e descreveram-se quatro casos de neoplasias relacionadas de câncer de endométrio e câncer colorretal. É possível que fatores genéticos e/ou hormonais estejam envolvidos no surgimento de neoplasias intestinais e extra-intestinais, especialmente de endométrio e ovário. Cabe ressaltar a importância da efetiva vigilância destas neoplasias em população de risco.
\end{abstract}

Palavras-chave: neoplasias colorretais; neoplasias dos genitais femininos; registros hospitalares; fatores de risco; neoplasias; prevenção e controle; epidemiologia; Brasil.

\footnotetext{
*Trabalho realizado no Registro de Câncer de Base Populacional de Campinas.

${ }^{1}$ M édica Reumatologista. Pós-graduanda em Saúde Coletiva (área de concentração em Epidemiologia) Faculdade de Ciências M édicas da U niversidade Estadual de Campinas (FCM -UN ICAM P). Coordenadora Executiva do Registro de Câncer de Base Populacional de Campinas. Enviar correspondência para N.M. UNICAM P - Prédio do CIPOI, Cidade Universitária Zeferino Vaz, Rua Vital Brasil 100, 30 Piso, Sala 08; 13083-970 Camoinas, SP - Brasil.

2Prof. Dr. Associado de Epidemiologia do D epartamento de M edicina Preventiva e Social - U N ICAM P. Coordenador do Registro de Câncer de Base Populacional de Campinas, SP - Brasil.

Recebido em setembro de 2001.
} 


\section{Abstract}

The population-based cancer registry is an important source of cases for cancer epidemiology and prevention studies. Colorectal cancer is the second cause of death from cancer in women in the United States. Weinberg et al, ${ }^{8}$ through the study of 101,734 cases of endometrial, cervical and ovarian cancers registered in the Surveillance, Epidemiology, and End Results (SEER) demonstrated the relative risk (RR) increase of colorectal cancers, following ovarian $(R R=3,7)$ and endometrial ( $R R=3,4)$ cancer. The population-based cancer registry of Campinas - São Paulo, based on 6,539 recorded cases of incident malignant neoplasmsin thearea between 1991 and 1995, used a methodologe similar to the one used in the above mentioned study to identify the cases of gynecologic cancer related to colorectal cancer, and collected 810 cases of women with diagnosis of cervical (265), endometrial (178), ovarian (97) and colorectal (270) cancer. In this study the mean age of pati ents with cervical cancer was 53.6; endometrial, 62.2; ovarian, 53.9 and colorectal, 61.9 years. After comparison of all selected cases, two patients were identified and four cancer cases associated to endometrial and colorectal cancer were described. It is possible that genetic or hormonal factors are responsible for the emergence of intestinal and extra-intestinal neoplasms, especially endometrial and ovarian. It is necessary to emphasize the importance of effective surveillance of this neoplasm in the population at risk.

Key words: colorectal neoplasms; female genital neoplasms, hospital records; risk factors, neoplasms, prevention and control; epidemiology; Brazil.

\section{INTRODUÇÃO}

0 câncer como patologia humana está registrado desde 1.600 a.C. em papiros do antigo Egito epermanece, como uma das mais importantes doenças, pela sua incidência e mortalidade, até os nossos dias. ${ }^{1}$

N os Estados Unidos, o câncer colorretal é a terceira causa de incidência e mortalidade em homens, depois do câncer de pulmão e próstata. $E$, também, é a terceira causa de incidência e mortalidade, depois do câncer de pulmão e mama, no sexo feminino. ${ }^{2-4}$

No Brasil, as taxas de mortalidade por câncer de cólon e reto apresentaram um acréscimo com variação percentual relativa de $65 \%$ para homens e $61 \%$ para mulheres no período de 1978 e $1998 .{ }^{5}$

Estudos epidemiológicos demonstraram 0 aumento de risco de câncer de cólon e reto de 1,5 a 3,0 em mulheres que tiveram, anteriormente, neoplasia maligna primária de endométrio ou ovário. ${ }^{6,7}$

Weinberg et al, ${ }^{8}$ quantificaram o risco de câncer de cólon e reto, posteriormente à neoplasia maligna do endométrio, colo uterino e ovário, a partir de casos registrados, no período de vinte um anos (1974 e 1995), no programa de vigilância e epidemiologia (Surveillance, Epidemiology, and End Results -
SEER - uma rede de nove registros de câncer de base populacional com área de cobertura de aproximadamente $10 \%$ da população dos Estados U nidos). N este intervalo de tempo foram registradas 118.505 mulheres com neoplasia maligna ginecológica. D esta coorte retrospectiva, excluíram-se os casos com diagnósticos realizados por autópsias e atestados de óbitos, além dos casos com ausência de informação do tempo de seguimento. Incluíram-se, somente, mulheres de etnia branca ou negra e idade superior a 25 anos, no momento do diagnóstico.

Assim, avaliou-se o risco de câncer colorretal em 101.734 casos de neoplasias de endométrio, colo uterino, ovário e as características étnicas, o estadiamento e 0 tempo de seguimento. Os autores identificaram, usando a mesma base de dados, os casos de mulheres com câncer de cólon e reto invasivo, subseqüentes à neoplasia ginecológica. Asmédias deidade, no momento do diagnóstico, foram de 49 anos para 0 câncer de colo do útero, 63 para endométrio e 61 para ovário. N o grupo de câncer de colo do útero predominaram as mulheres da raça negra. Q uanto ao seguimento, comparativamente, as pacientes com neoplasia de ovário, tiveram em média, dois anos, as mulheres com neoplasia de colo do útero, 
cinco anos, e as pacientes com câncer de endométrio foram acompanhadas, em média, durante sete anos.

Feito o ajuste para idade, etnia e estadiamento, o modelo de regressão, mostrou que os cânceres de ovário e endométrio apresentaram um aumento de risco para a neoplasia de cólon e reto, porém, o mesmo não ocorreu para a neoplasia de colo do útero. D esta forma, o risco relativo (RR) de câncer colorretal, posterior ao câncer de ovário, foi maior para faixa etária de 25 a 49 anos (RR = $3,67$ ) e no grupo de 50 a 64 anos ( $R R=1,52)$, decrescendo, no grupo de mulheres com mais de 65 anos ( $R R=1,03)$. N os casos de câncer de endométrio o risco relativo para o câncer colorretal foi de 3,39 para as mulheres com menos de 50 anos.

A propósito desde estudo8 e da revisão da literatura ${ }^{9-13}$ o Registro de C âncer de Base Populacional de Campinas-SP (RCBP( ampinas-SP), analisou 1.024 casos de mulheres registradas no período de cinco anos (1991 a 1995) com neoplasias primárias de colo do útero, ovário, endométrio, cólon e reto, para identificar casos de câncer ginecológico relacionados com câncer colorretal e vice-versa, neste subgrupo de casos registrados. $E$, foi encontrado, nessa base de dados, em aproximadamente, cinco anos de seguimento, um caso de câncer de endométrio com subseqüente neoplasia maligna de cólon. E em contrapartida, ao realizar-se o estudo no sentido inverso, isto é, partindo-se do diagnóstico de câncer de cólon e a sua relação com neoplasia maligna ginecológica, na mesma base de dados, encontrou-se, em cerca de quatro anos de seguimento, um outro caso de câncer de cólon, que contrariamente, teve como subseqüente neoplasia maligna de endométrio.

0 objetivo deste estudo é demonstrar que, mesmo em uma base de dados numericamente pequena como é a da RCBPCampinas-SP $(6.539$ casos incidentes consolidados), quando comparada a outros registros de câncer de base populacional nacionais e internacionais, é possível identificar os casos de câncer ginecológico relacionados com o câncer colorretal e viceversa, na população de cobertura do registro de câncer de base populacional evidenciando- se a importância da vigilância efetiva, dessas neoplasias na população de risco.

\section{PACIENTES E MÉTODOS}

0 Registro de Câncer de Base Populacional de Campinas-SP (RCBP-Campinas-SP) existe desde 1991, foi criado pela lei municipal 6.986, de 11 de maio de 1992.

Coleta todos os casos novos de câncer ano a ano do M unicípio de Campinas-SP, arquiva, analisa as informações e divulga os dados consolidados para a comunidade médica e autoridades em saúde.

O RCBP-Campinas-SP tem como área de cobertura geográfica $794 \mathrm{Km}^{2}$ e população de 908.906 habitantes (contagem populacional de 1996- IBGE), com $97 \%$ da população em área urbana e o crescimento anual de $1,43 \%$.

O s casos do RCBP-C ampinas-SP são registrados de acordo com as padronizações nacionais do Instituto $\mathrm{N}$ acional de $\mathrm{Câncer}$ (IN CA $)^{14}$ e internacionais da Agência Internacional para Pesquisa em Câncer (IARC ) ${ }^{15-17}$ para informações coletadas, codificação, classificação (Classificação Internacional de D oenças para O ncologiaCID - ${ }^{18,19}$ e Classificação Internacional de D oenças-CID ), ,20,21 validação, consistência, análise, confidencialidade e divulgação dos dados.

N o período de 1991 a 1995, registram-se 6.539 casos incidentes de neoplasias malignas primárias de pessoas residentes em cinco distritos do Município de Campinas, a saber, Joaquim Egídio, Sousas, N ova Aparecida, Barão Geraldo e Campinas. A partir, desta base de dados, atualizada com os dados de mortalidade até 0 ano de 2.000, reuniram-se 1.024 casos de mulheres com diagnóstico de neoplasias malignas do colo do útero, endométrio, ovário, cólon e reto e idade superior a 25 anos, no momento do diagnóstico.

Excluíram-se 214 casos (20,9\%), sendo $156(15,2 \%)$ casos de neoplasias malignas in situ, $53(5,2 \%)$ casos com diagnósticos realizados somente através dos atestados de óbitos (D CO ), e 5 (0,5\%) casos dediagnósticos por meio de autópsia.

Assim, analisaram-se 810 casos de neoplasias malignas, sendo 540 casos de 
câncer de colo do útero, endométrio, ovário, para verificar se havia neoplasia colorretal secundária e, inversamente, se havia neoplasia ginecológica nos 270 casos de câncer de cólon e reto estudados, constatantes de um banco de dados construído no programa EPIIN FO, 22 bem como, as variáveis: idade, cor da pele, extensão da neoplasia e verificação histológica.

\section{RESULTADOS}

A Tabela 1 mostra a freqüência de distribuição das características selecionadas nos 810 casos observados para identificação de neoplasia ginecológica relacionadas com câncer de cólon e reto, sendo 265 (32,7\%) cânceres de colo de útero, $178(22 \%)$ de endométrio, $97(12 \%)$ de ovário e 270 $(33,3 \%)$ de cólon e reto. A média de idade para câncer de colo do útero foi de 53,6 anos; endométrio 62,2; ovário 53,9 e colorretal 61,9 anos. A cor da pele, predominante em todos os tipos de cânceres foi a branca, em média $64,7 \%$, embora $28,9 \%$ dos casos não tivessem essa. Q uanto à extensão da neoplasia 22,5\% estavam localizadas; 18,2\% tinham metástases regionais (linfonodos); 8,7\% apresentavam metástases a distância, porém, em 50,9\% dos casos não foi possível, obter esta informação, dado que os registros de câncer de base populacional, por sigilo e confidencialidade, não tem acesso direto ao paciente, somente, às fontes de informação (prontuários, laudos da anatomia patológica e outros). N 0 entanto, a retirada destes casos da base de dados reduziria a casuística em cerca de $50 \%$. Por outro lado, a verificação microscópica (histologia), após as exclusões foi realizada em 97,3\% dos casos com diagnóstico do tumor primário, em média.

Tabela 1. Distribuição das características selecionadas de mulheres com neoplasias malignas registradas no RCBP-Campinas-SP (n=810), no período de 1991-1995, Campinas, São Paulo

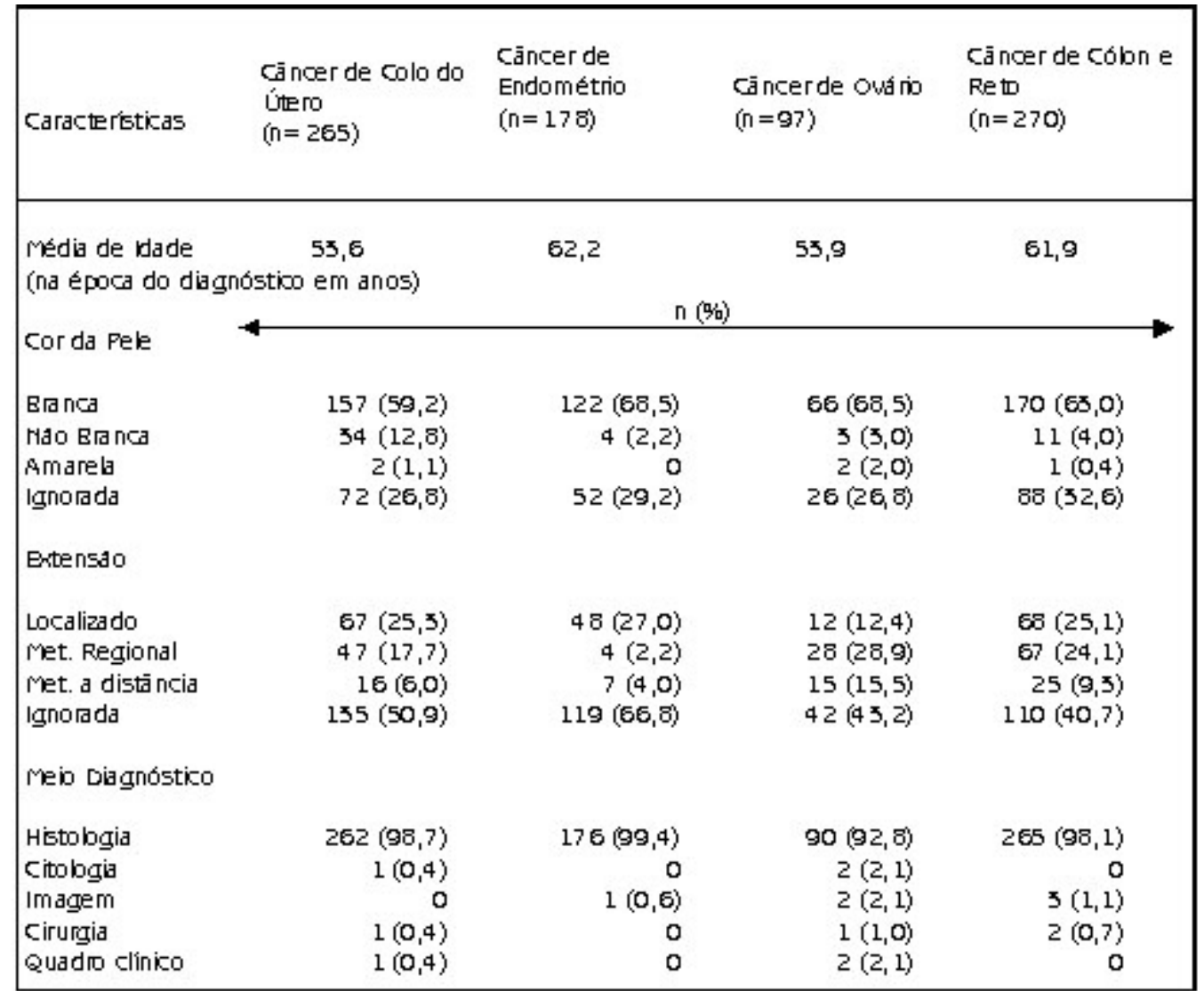




\section{DESCRIÇÃO DOS CASOS}

As duas primeiras neoplasias encontradas foram, da paciente E.G.M, sexo feminino, 70 anos, cor branca, natural de Santa Rita, São Paulo, nascida em 25/02/1921, aposentada, residente em $C$ ampinas. A data do diagnóstico da primeira neoplasia primária (endométrio) foi em 15/04/1991, com a seguinte descrição do exame histológico: "Adenocarcinoma bem diferenciado do endométrio com diferenciação escamosa. Infiltrativo para o miométrio até o terço médio da espessura miometrial ecom extensão endocervical. O vários atróficos. $\mathrm{H}$ idátide de M orgagni em trompas."

0 câncer foi codificado e registrado no RCBP-Campinas-SP como T-182.0 (topografia) ${ }^{18}$ e M-8140/3 (morfologia), ${ }^{18}$ através da CID 9-0 ${ }^{18}$ em 1991. A data do diagnóstico da segunda neoplasia (cólon ascendente), não metastática da primeira, foi 26/12/1995, o laudo do exame histológico relatou: "Adenocarcinoma pouco diferenciado em cólon ascendente. Infiltração do tecido adiposo pericólico. M etástase para 4 de 10 linfonodos". O caso foi codificado e registrado no $\mathrm{RCBP}-\mathrm{C}$ ampinas-SP como C 18.2 (topografia) ${ }^{19} \mathrm{eM}-8140 / 33$ (morfologia), ${ }^{19}$ através da CID 10-0-2 ${ }^{19} \mathrm{em}$ 1995. A paciente faleceu em 28/06/1997 com diagnóstico no atestado de óbito de infecção do trato urinário de localização não especificada (CID -10 = N 39.0). ${ }^{21}$

Os outros dois casos encontrados na mesma base de dados selecionada foram da paciente N.A.O.S, sexo feminino, 41 anos, cor branca, natural de Campinas, São Paulo, nascida em 20/09/1949, cabeleireira, residente em $C$ ampinas. $A$ data do primeiro diagnóstico da neoplasia primária (cólon transverso) foi em 05/02/1991, com a seguinte descrição do exame do exame histológico: "Adenocarcinoma bem diferenciado mucosecretor de cólon transverso com invasão da camada serosa, ulcerada e perfurada. Linfonodos (5) sem acometimento de neoplasia".

0 câncer foi codificado e registrado no RCBP-Campinas-SP como T-153.1 (topografia) ${ }^{18}$ e M -8481/3 (morfologia) ${ }^{18} \mathrm{em}$ 1991. A data do segundo diagnóstico de neoplasia primária (endométrio) foi em 15/12/ 1994, onde o relato do exame histológico descrevia: "Adenocarcinoma do endométrio moderadamente diferenciado infiltrativo em miométrio". Estadiamento IIb. A neoplasia foi codificada e registrada do RCBPCampinas-SP como C 54.1 (topografia) ${ }^{19} \mathrm{eM}$ 8140/32 (morfologia) ${ }^{19} \mathrm{em}$ 1994. A paciente foi a óbito em 30/01/1997 com o diagnóstico no atestado de óbito de neoplasia maligna do corpo do útero $(\mathrm{CID}-10=\mathrm{C} 54.1) .{ }^{21}$

\section{DISCUSSÃO}

Em geral os estudos de risco relativo para cânceres secundários são realizados de forma unilateral. ${ }^{6-8,23-29} \mathrm{~N}$ o entanto, na base de dados selecionada do RCBP-Campinas-SP, a identificação dos casos de câncer ginecológico relacionados com o câncer colorretal realizouse nos dois sentidos, e os casos encontrados, provavelmente, têm etiologias diferentes. Em outras palavras, identificou-se a neoplasia ginecológica (câncer de endométrio) tanto procedendo (caso da primeira doente), quanto sucedendo (caso da segunda paciente) câncer de cólon, após cuidadosa revisão e um período de observação de no mínimo três anos e meio, considerando-se que as recidivas surgem, freqüentemente, nos dois primeiros anos subseqüentes ao diagnóstico do câncer primário. ${ }^{30,31} \mathrm{Um}$ número elevado de casos não registrava informação sobre cor da pele ( $26,8 \%$ ) e extensão da doença ( $50,9 \%$ ), o que demonstra que a informação no prontuário do doente, precisa ser melhorada para que os estudos sejam conclusivos. Entretanto, em média, 97,3\% dos diagnósticos dos casos selecionados foram confirmados por meio da verificação histológica.

0 aumento do risco relativo da ocorrência de outros cânceres secundários ao câncer primário está relatado para diferentes populações; ${ }^{8,23-27}$ contudo, os fatores de risco específicos para esses cânceres são desconhecidos. Muitas neoplasias endometriais ocorrem após os 50 anos (na menopausa), em mulheres sem a síndrome familiar de câncer colorretal, e os fatores genéticos envolvidos nestes casos ainda não são claros. Existem poucos estudos para investigação da história familiar de câncer como fator de risco para neoplasia de endométrio na mulher idosa. ${ }^{32}$ Alguns 
pesquisadores sugerem a interação de fatores nutricionais e hormonais na gênese de cânceres secundários ginecológicos e de outras topografias;33 porém, são necessários novos estudos para consolidar as conclusões.

Cabe lembrar que o primeiro caso identificado na base selecionada do RCBPC ampinas-SP foi de uma paciente que teve câncer de endométrio aos 70 anos de idade (menopausada) e neoplasia de cólon, quatro anos e sete meses depois. Infelizmente, não foi possível, obter-se a história familiar de câncer desta paciente.

Por outro lado, sabemos que o câncer colorretal não polipóide faz parte da síndrome familiar, caracterizada pelo desenvolvimento precoce de câncer colorretal e câncer extraintestinal, particularmente, o câncer de endométrio e ovário. N esta síndrome hereditária, a neoplasia colorretal, ocorre na faixa etária dos 40 a 45 anos, o câncer de endométrio acomete $40 \%$ das mulheres e a neoplasia de ovário, $10 \%{ }^{8,34}$

Como podemos observar, a segunda paciente identificada na base selecionada do RCBP-C ampinas-SP tevecâncer colorretal aos 41 anos de idade e diagnóstico de câncer de endométrio três anos e sete meses depois. $\mathrm{N}$ este caso, também não foi possível, conhecermos a história familiar e, conseqüentemente, não temos informação para concluir se este caso estava relacionado à síndrome familiar de câncer colorretal não polipóide ou não.

Assim, o esclarecimento dos mecanismos da etiologia da associação de múltiplas neoplasias requer mais estudos elucidativos em vários níveis: molecular, histológico, imunológico, clínico, epidemiológico, entre outros, através de casos de fontes qualificadas, ressaltando que os estudos internacionais utilizam, cada vez mais, como fonte de casos, os Registros de Câncer de seus países. ${ }^{7-8,23-25,28,35}$

\section{CONCLUSÕES}

0 número de casos de câncer ginecológico relacionados com o câncer colorretal e vice versa pode ser identificado e quantificado no B rasil através dos Registros de $C$ âncer de Base Populacional (RCBPs), para as mais diferentes populações, delimitadas pelas áreas geográficas de abrangência destes registros. 0 grande número de casos dos RCBPs minimiza os vieses de seleção das séries de casos dos hospitais e das clínicas especializadas em O ncologia. Por outro lado, os Registros H ospitalares de Câncer (RHC) podem, igualmente, contribuir de forma decisiva, devido a maior acessibilidade ao doente, através do seu seguimento no hospital, fato que possibilita que as informações individualizadas do paciente sejam mais completas e acuradas, especialmente, os seus dados de antecedentes pessoais, ocupacionais e familiares, sua história clínica e sua evolução, permitindo a realização de estudos epidemiológicos analíticos orientados para 0 esclarecimento das questões etiológicas e de fatores de risco específicos desconhecidos na relação do câncer ginecológico com o câncer colorretal, dentre outras associações. 0 utro fato, não menos importante é que as informações obtidas através dos R egistros de Câncer possibilitam maior ênfase na prevenção e no planejamento adequado de programas de vigilância do câncer ginecológico e do câncer colorretal em população de risco no nosso meio.

\section{AGRADECIMENTOS}

Agradecemos a Sra. Sônia Pereira Ramos, registradora de câncer do Registro de Câncer de Base Populacional de Campinas - São Paulo, pela sua competência e dedicação na coleta e digitação dos casos de neoplasias do município de Campinas.

\section{REFERÊNCIAS BIBLIOGRÁFICAS}

1. N ational Cancer Institute(US). Closing in on cancer solving a 5000-year-old mystery. Bethesda: N Cl; 1998. (N IH Publication; no. 98-2955).

2. Ries $L A G, W$ ingo $P A, M$ iller $D D$, et al. The annual report to the nation on the status of cancer, 1973-1997, with a special section on colorectal cancer. Cancer 2000; 88(10):2398424.

3. Greenlee RT, M urray T, Bolden $\mathrm{S}$, W ingo PA. Cancer statistics 2000. CA C ancer J Clin 2000;50(1):7-33. 
4. Pisani P, Parkin D M , Bray F, et al. Estimates of the wordwide mortality from 25 cancers in 1990. Int J Cancer 1999;83:18-29.

5. Instituto $\mathrm{N}$ acional deCâncer (Brasil). Estimativas deincidênciaemortalidadepor câncer no Brasil 2001. Rio deJaneiro: IN CA: 2001.

6. Bergfelot K, Einhorn S, Rosendahl I, et al. Increased risk of second primary malignanciesin patients with gynecological cancer. A swedish record-linkage study. Acta O ncol 1995;34(6):771-7.

7. Travis LB, CurtisRE, Boice JD Jr, Platz CE, $H$ ankey BF Jr. Second neoplasm among longterm survivors of ovarian cancer. $C$ ancer Res 1996;56:1564-70.

8. Weinberg DS, N ewschaffer CJ, Topham A. Risk for colorectal cancer after gynecologic cancer. Ann Intern M ed 1999;131:189-93.

9. D auplat J, Bignon Y, Pomel $C$, et al. Search for genetic risk in surgical indicationsin oncology. Press M ed 1996;25(34):1633-5.

10. Schoen RE, Weissfeld JL, Kuller LH . Arewomen with breast, endometrial or ovarian cancer at increased risk for colorectal cancer? Am J Gastroenterol 1994;89:835-42.

11. Eisen GM , Sandler RS. Arewomen with breast cancer morelikely to develop colorectal cancer? Critical review and meta-analysis. J Clin Gastroenterol 1994;19:57-63.

12. N elson CL, SellersTA, Rich SS, et al. Familial clustering of colon, breast, uterine, and ovarian cancers as assessed by family history. Genet Epidemiol 1993; 10:235-44.

13. Reimer RR, H oover R, FraumeriJF Jr, YoungRC. Second primary neoplasmas following ovarian cancer. J N atl C ancer Inst 1978;61:1195-7.

14. Secretaria $N$ acional de Assistência à Saúde. Instituto $\mathrm{N}$ acional de $\mathrm{C}$ âncer. Coordenadoria deProgramas de Controledo C âncer Pro-O nco. (Brasil). Registro de câncer: princípios e métodos. Rio de Janeiro: IN CA; 1995.

15. Jensen $O M$, Parkin $D M, M$ acLennan $R, M$ uir $C S$, Skeet $R G$, organizers. $C$ ancer registration principles and methods. Lyon: International Agency for Research on C ancer; 1991. (IARC Technical Report; no. 95).

16. Parkin D M , Chen VW, Ferlay J, G alceran J, Storm H H, W helan SL. Comparability and quality control in cancer registration. Lyon: Internacional Agency for R esearch on C ancer; 1994. (IARC Technical Report; no. 19).

17. Parkin D M , W helan SL, Ferlay J, Raymond L, Young J, editors. C ancer incidencein fivecon- tinents. Lyon: International Agency for Research on Cancer; 1997. vol.7 (IARC Scientific Publications; no.143).

18. M inistério da Previdência eAssistência Social (Brasil). Classificação Internacional deD oenças para O ncologia. Revisão de 1975. Rio de Janeiro: D ATAPREV; 1989.

19. Percy C, Van Holten V, M uir C, editors. Classificação Internacional de D oenças para Oncologia CID-0. 2a ed. São Paulo: Universidade de São Paulo. Fundação Oncocentro; 1996.

20. M inistério da PrevidênciaeAssistência Social (Brasil). Classificação Internacional deD oenças. Revisão de1975. Rio deJ aneiro. D ATAPREV; 1980.

21. 0 M S. C lassificação estatística internacional de doençase problemas relacionadosàsaúde. CID 10. 5a ed. São Paulo: U niversidadedeSão Paulo; 1997.

22. D ean AG, D ean JA, C oulombier D, et al. EpiInfo [computer program]. Version 6: a word processing database, and statistics program for epidemiology on microcomputers. Atlanta (GE): C enters of D iseaseC ontrol and Prevention; 1994.

23. M cC redieM , M cfarlaneG J, Bell J, C oates M . Second primary cancers after cancers of the coIon and rectum in N ew South Wales, Australia, 1972-1991. Cancer Epidemiol BiomarkesPrev 1997;6:155-60.

24. H oar SK, W ilson J, Blot WJ, M cLaughlin JK, Winn D M , Kantor AF. Second cancer following cancer of the digestive system in Connecticut, 1935-82. N CI M onogr 1985;68:49-82.

25. Lynge $E$, Jensen $O M, C$ arstensen $B$. Second cancer following cancer of the digestivesystem in Denmark, 1943-80. N Cl M onogr 1985;68:277-308.

26. TanakaH , H iyamaT, H anai A, Fujimoto I. Second primary cancersfollowing colon and rectal cancers in 0 saka, Japan. Jpn J Cancer Res 1991;82:1356-65.

27. Teppo L, PukkalaE, Saxén E. M ultiplecancer: an epidemiologic exercise in Finland. J $\mathrm{N}$ atl Cancer Inst 1985;75:207-17.

28. Enblad P, Adami H -O , G limeliys B, Krusemo $U$, Pahlman $L$. T he risk of subsequent primary malignant diseases after cancers of the colon and rectum. Cancer 1990;65:2091-100.

29. M cCredie M, M acfarlane GJ, Coates M S, O sborn RA. Risk of second malignant neoplasmsfollowing femalegenital tract cancersin 
N ew South Wales (Australia), 1972-1991. Int J Gynecol Cancer 1996;6:362-68.

30. Pahlman L, G limeliusB. Local recurrenceafter surgical treatment for rectal carcinoma. Acta Chir Scand 1984;150:331-35.

31. Floyd CE, Corley RG, C ohn I. Local recurrence of carcinoma of the colon and rectum. Am J Surg 1965;109:153-59.

32. O Ison JE, SelleresTA, Anderson KE, Folsom AR. D oesafamily history of cancer increasethe risk for postmenopausal endometrial carcinoma? A prospective cohort study and a nested casecontrol family study of older women. Cancer
1999;85(11):2444-9.

33. SellersTA, Kushi LH, Potter JD, et al. Effect of family history, body-fat distribution, and reproductivefactorson therisk of postmenopausal breast cancer. N Engl J M ed 1992;326:1323-9.

34. Watson P, Lynch H T. Extracolonic cancer in hereditary nonpolyposiscolorectal cancer. Cancer 1993;71(3):677-85.

35. Kaldor JM, D ay NE, Band P, et al. Second malignancies following testicular cancer, ovarian cancer and H odgkin's disease: an international collaborative study among cancer registries. Int J C ancer 1987;39:571-85. 